\title{
Análise da atuação fisioterapêutica nas sequelas motoras da Síndrome de Guillain-Barré: uma revisão bibliográfica
}

A Síndrome de Guillain-Barré é uma polineuropatia desmielinizante que acomete o Sistema Nervoso Periférico (SNP), de etiologia desconhecida, e que ocasiona fraqueza motora ascendente. Tem como principais manifestações clínicas a parestesia, ou paralisia distal; alteração da sensibilidade tátil, dolorosa e proprioceptiva; fraqueza muscular; alterações posturais; comprometimento da marcha; e em casos graves, pode evoluir para insuficiência respiratória; taquicardia sinusal; variações de pressão arterial; e retenção urinária, podendo surgir ainda sudorese profunda, e em decorrência desses, o paciente pode necessitar de ventilação mecânica. A literatura descreve tratamentos fisioterapêuticos de maneiras distintas, sendo que cada fisioterapeuta com sua metodologia visa um objetivo em comum, que é o de promover e melhorar de forma geral a qualidade de vida dos pacientes acometidos pela Síndrome de Guillain-Barré. Os resultados adquiridos neste estudo demonstram que não existe um protocolo específico de tratamento na Síndrome de Guillain-Barré, sendo que os dados obtidos não podem ser generalizados. Portanto, é necessário a realização de estudos a respeito da atuação fisioterapêutica na Síndrome Guillain-Barré, afim de se criar intervenções de reabilitação que visem melhorar a qualidade de vida desses pacientes.

Palavras-chave: Síndrome de Guillain-Barré; Fisioterapia; Reabilitação.

\section{Analysis of the physiotherapeutic performance in the motor sequelae of Guillain-Barré Syndrome: a bibliographic review}

Guillain-Barré Syndrome is a demyelinating polyneuropathy that affects the Peripheral Nervous System (SNP) of unknown etiology and causes ascending motor weakness. Its main clinical manifestations are paresthesia, or distal paralysis; alteration of tactile, painful and proprioceptive sensitivity; muscle weakness; postural changes; gait impairment; and in severe cases, it may progress to respiratory failure; sinus tachycardia; changes in blood pressure; and urinary retention, and deep sweating may still occur, and as a result, the patient may require mechanical ventilation. The literature describes physiotherapeutic treatments in different ways, and each physiotherapist with his methodology aims at a common goal, which is to promote and improve in general the quality of life of patients affected by Guillain-Barré syndrome. The results obtained in this study demonstrate that there is no specific treatment protocol in Guillain-Barré syndrome, and the data obtained can not be generalized. Therefore, it is necessary to carry out studies regarding the physical therapy in Guillain-Barré Syndrome, in order to create rehabilitation interventions aimed at improving the quality of life of these patients.

Keywords: Guillain-Barré Syndrome; Physiotherapy; Rehabilitation.

Topic: Fisioterapia

Reviewed anonymously in the process of blind peer
Received: 14/12/2017

Approved: 23/12/2017
Paula Roberta Ferreira Costa (iD)

Faculdade Guaraí, Brasil

http://lattes.cnpq.br/8946206550686612

http://orcid.org/0000-0001-9731-9808

paulacosta_sol@hotmail.com

Chirley Silva Rodrigues

Faculdade Guaraí, Brasil

http://lattes.cnpq.br/5955946582624813

http://orcid.org/0000-0002-1958-3555

chirlleypgd@hotmail.com

Karla Camila Correia da Silva

Faculdade Guaraí, Brasil

http://lattes.cnpq.br/1981447087125364

karlacamilac@yahoo.com.br

\section{Referencing this:}

COSTA, P. R. F.; RODRIGUES, C. S.; SILVA, K. C. C.. Análise da atuação fisioterapêutica nas sequelas motoras da Síndrome de Guillain-Barré: uma revisão bibliográfica. Scire Salutis, v.7, n.2, p.42-49, 2017. DOI: http://doi.org/10.6008/SPC2236-9600.2017.001.0005 


\section{INTRODUÇÃO}

A Síndrome de Guillain-Barré (SGB) é uma polineuropatia inflamatória desmielinizante aguda que acomete o Sistema Nervoso Periférico (SNP), ocasionando fraqueza motora ascendente, progredindo para os membros superiores, face, músculos respiratórios e alterações sensoriais resultante da perda de reflexos tendinosos profundos (FERRANINI et al., 2011).

Rodrigues (2015) enfatiza que a SGB é uma doença de caráter autoimune, onde ocorre a degeneração e/ou desmielinização da bainha de mielina manifestando uma inflamação aguda dos nervos e, às vezes, das raízes nervosas (SNP). Este processo interfere na condução de sinais advindo do sistema nervoso até a musculatura, e em parte dos casos, no sentido contrário, isto é, na condução dos estímulos sensoriais até o cérebro, levando a uma perda da habilidade de grupos musculares responderem aos comandos cerebrais. (SNC) O cérebro também recebe menos sinais sensitivos do corpo, resultando em inabilidade para sentir o contato com a pele, dor ou calor.

Montini et al. (2016) relatam que a etiologia da SGB ainda não foi identificada, porém pode ser observada uma relação com doenças causadas por vírus e bactérias, sendo o mais frequente a bactéria Campylobacter Jejuni. Em dois terços dos casos possuem relatos de infecção respiratória ou gastrointestinais nas seis semanas que precederam o quadro.

Segundo Quadros (2017, citado por VAN DOORN, 2008) a SGB tem progressão rápida, e seu pico degenerativo pode acontecer em até quatro semanas após o início dos sintomas. Devido acometimento do sistema nervoso periférico (SNP), a SGB tem como principais manifestações clínicas a parestesia, ou paralisia distal, alteração da sensibilidade tátil, dolorosa e proprioceptiva, fraqueza muscular, alterações posturais, comprometimento da marcha. Santos et al. (2017, citados por NETO et al., 2014) afirmam que em casos mais graves podem evoluir para insuficiência respiratória, taquicardia sinusal e variações de pressão arterial, retenção urinária, podendo surgir ainda sudorese profunda e em decorrência desses, o paciente pode necessitar de ventilação mecânica.

Segundo a Organização Mundial da Saúde, os dados do sistema de vigilância hospitalar revelam que houve um aumento considerável de casos da Síndrome de Guillain-Barré no Brasil: entre janeiro e novembro de 2015, 1708 (um mil setecentos e oito) casos novos em todo o país. Moraes et al. (2015) relata que, a princípio, o diagnóstico da SGB é realizado através do quadro clínico onde o paciente apresenta dificuldade ao deambular, dormência ou fraqueza na musculatura, perda de força e em alguns casos dor em toda extremidade corpórea. Após a confirmação do quadro clínico descrito o paciente deverá realizar o diagnóstico laboratorial onde é colhido o líquido cefalorraquidiano para análise ou através do exame de eletroneuromiografia.

A fisioterapia na SGB contribui para diminuição do tempo de recuperação do paciente e na prevenção das complicações que levam a este necessitar de uma ventilação mecânica invasiva ou não invasiva, contribuindo na diminuição da mortalidade. A reabilitação motora é essencial na minimização e reabilitação 
das sequelas motoras, promovendo independência e melhorando a qualidade de vida do paciente. (SOARES, et al., 2017, citado por LEILA et. al., 2016; TUACEK et al., 2012).

Esta revisão de literatura teve como objetivos: descobrir como a fisioterapia auxilia o paciente com a SGB; investigar quais os benefícios que a fisioterapia proporciona ao paciente com a SGB; analisar quais os objetivos da fisioterapia no paciente com SGB; verificar como é a abordagem da fisioterapia no paciente com a SGB; e descobrir quais os métodos e técnicas fisioterapêuticas utilizadas para o tratamento das sequelas da SGB.

\section{METODOLOGIA}

A presente pesquisa trata de uma revisão de literatura que busca demonstrar a contribuição da fisioterapia na recuperação do paciente portador da Síndrome de Guillain-Barré. A coleta de dados foi realizada no período de setembro a novembro de 2017, selecionando-se livros e artigos em conformidade com o assunto proposto. Para isso, realizou-se um levantamento bibliográfico nas bases de dados eletrônicas: Biblioteca Virtual em Saúde (BVS), Physiotherapy Evidence Database (PEDro), e Scientific Eletronic Library Online (SciELO). Foram utilizados os seguintes descritores: Síndrome de Guillain-Barré; Fisioterapia; e Reabilitação.

A pesquisa incluiu apenas estudos publicados no período de 2010 a 2017, e limitou-se aos idiomas português, inglês e espanhol, sendo excluídos todos os artigos incompletos, resumos, bem como aqueles que não possuem fundamentação cientifica e/ou que não estiveram relacionados ao tema proposto. Com o auxílio dos descritores encontrou-se 30 artigos. Após a aplicação dos filtros: data de publicação (2008-2017), idiomas (inglês, português e espanhol) e textos completos, e após análise criteriosa dos achados, selecionouse uma quantidade de 25 periódicos para a confecção do presente trabalho.

\section{RESULTADOS E DISCUSSÃO}

O tratamento fisioterapêutico na síndrome de Guillain-Barré pode ser realizado por diferentes condutas, cabendo ao fisioterapeuta identificar as principais complicações de cada paciente, e adaptá-los ao tratamento proposto. No quadro 1 , relatamos as condutas realizadas e os resultados obtidos dentro da literatura estudada.

Quadro 1: Objetivos do tratamento fisioterapêutico no paciente portador da síndrome de Guillain-Barré.

\begin{tabular}{|c|c|c|}
\hline AUTOR E ANO & CONDUTAS REALIZADAS & RESULTADOS \\
\hline $\begin{array}{l}\text { CARVALHO, } \\
\text { T.G.M.L.; LOPES, R. } \\
\text { C., } 2013 .\end{array}$ & $\begin{array}{l}\text { Cinesioterapia exercícios passivos associado a termoterapia } \\
\text { (infravermelho), alongamentos globais, exercícios metabólicos, } \\
\text { técnica de co-contração, hidroterapia (turbilhão MMSS e MMII), } \\
\text { treino de transferências equilíbrio, exercícios resistidos em } \\
\text { cadeia cinética fechada e aberta, readequação a função de } \\
\text { marcha. }\end{array}$ & $\begin{array}{l}\text { Independência completa, aumento de todas as } \\
\text { funções motoras (força, tônus muscular e } \\
\text { coordenação), respostas normais de MMSS, MMII e } \\
\text { tronco, total independência nas AVDs. }\end{array}$ \\
\hline $\begin{array}{l}\text { SÁ, B.P, GRAVE, M. } \\
\text { T. Q; PÉRICO, E.; } \\
\text { BOHRER, T. R. M. J., } \\
\text { 2015. }\end{array}$ & $\begin{array}{l}\text { Alongamentos e reforço da musculatura dos MMII, método } \\
\text { Bobath: transferência e suporte de peso para os MMII, co- } \\
\text { contração, tapping de deslizamento e tapping alternado, método } \\
\text { PNF: iniciação rítmica, reversão dinâmica, reversão de } \\
\text { estabilização e estabilização rítmica. }\end{array}$ & $\begin{array}{l}\text { Progresso na força muscular de MMII, melhora na } \\
\text { ADM e equilíbrio. }\end{array}$ \\
\hline $\begin{array}{l}\text { MONTINI, F. T.; } \\
\text { SOUZA, D. R.; }\end{array}$ & $\begin{array}{l}\text { Alongamentos de membros inferiores, treino de transferências } \\
\text { com tábua, ortostatismo assistido em prancha, stand in table e }\end{array}$ & $\begin{array}{l}\text { Apresentou melhora do controle de tronco com } \\
\text { dissociação de cintura escapular, ganho de força e }\end{array}$ \\
\hline
\end{tabular}




\begin{tabular}{|c|c|c|}
\hline $\begin{array}{l}\text { RIBEIRO, F. Q; } \\
\text { BATTISTELLA, L. R., } \\
2016 .\end{array}$ & $\begin{array}{l}\text { barras paralelas (BP) com calha e tala extensora, trocas posturais } \\
\text { tais como decúbito ventral (DV) com cunha, decúbito lateral } \\
\text { esquerdo e direito, rolar; fortalecimento de musculatura de } \\
\text { tronco e abdominal em sedestação, DV e associado ao FES, além } \\
\text { do uso de ciclo ergômetro de membros superiores (MMSS) e } \\
\text { inferiores. }\end{array}$ & $\begin{array}{l}\text { melhora da coordenação em membros superiores, } \\
\text { conseguindo manter-se em ortostatismo no stand in } \\
\text { table e prancha ortostática sem sinais ou sintomas de } \\
\text { hipotensão postural e, quando em BP, conseguiu } \\
\text { manter-se ereto e retirar alternadamente as mãos } \\
\text { esboçando força para completar o passo. }\end{array}$ \\
\hline $\begin{array}{l}\text { NASCIMENTO, V. L. } \\
\text { S.; BORBA, G. S.; } \\
\text { LEITE, C. M. B.; } \\
\text { GABARINI, M. C., } \\
2012 .\end{array}$ & $\begin{array}{l}\text { Hidrocinesioterapia (Corrida estática e exercícios de polichinelo, } \\
\text { Corrida estática com pernas elevadas; corrida dinâmica através } \\
\text { do comprimento da piscina alongamento global na musculatura } \\
\text { dos membros superiores (MMSS) e dos membros inferiores } \\
\text { (MMII)). exercícios gerais e específicos (treino de transferências } \\
\text { de peso; estabilização do tronco (Bad Ragaz); exercícios } \\
\text { abdominais em diferentes decúbitos, exercícios em decúbitos, } \\
\text { agachamentos; chutes laterais; exercícios abdominais em } \\
\text { diferentes decúbitos, Treino de mudança de decúbitos + } \\
\text { polichinelo; agachamentos; chutes laterais e posteriores; treino } \\
\text { de escadas + resistência em MMSS; treino de bike com tronco } \\
\text { ereto; exercícios abdominais em diferentes decúbitos; treino de } \\
\text { encaixe e desencaixe do quadril, relaxamento Watsu, tração } \\
\text { cervical }\end{array}$ & $\begin{array}{l}\text { Ocorreu recuperação total das amplitudes dos } \\
\text { movimentos articulares, da força muscular e das } \\
\text { pressões inspiratória e expiratória em três meses de } \\
\text { tratamento. A melhora funcional também foi } \\
\text { percebida conforme os resultados nos diferentes } \\
\text { domínios do SF-36. Sem sequelas a paciente seguiu } \\
\text { normalmente sem recidiva do quadro após obter alta } \\
\text { fisioterapêutica. }\end{array}$ \\
\hline $\begin{array}{l}\text { QUADROS L.R, } \\
\text { GRAVE M.T.Q }\end{array}$ & $\begin{array}{l}\text { Em sedestação, transferência de peso entre membros, } \\
\text { flexionando o quadril contralateral - Transferência de peso em } \\
\text { sedestação sobre bola suíça - Passar de sedestação para } \\
\text { ortostase, transferindo todo o peso para o membro inferior } \\
\text { direito.- Retroversão do quadril e retificação lombar, em } \\
\text { sedestação sobre bola suíça - Flexão de tronco levando bola } \\
\text { suíça à frente, e retornando à posição inicial "enrolando" a } \\
\text { coluna - Ponte em quatro apoios, paciente realiza cifose de toda } \\
\text { a coluna, contraindo abdômen - Exercícios abdominais- } \\
\text { Aplicação de bandagem rígida - Flexão de dedos e arco plantar } \\
\text { dos pés, juntando uma toalha no chão. - Contração excêntrica de } \\
\text { quadríceps, passando da posição ortostática para sedestação } \\
\text { lentamente-Abdução e rotação externa de quadril resistida por } \\
\text { faixa elástica; - Passar de sedestação para ortostase com faixa } \\
\text { elástica na região dos joelhos, resistindo para abdução do } \\
\text { quadril - Extensão de joelho resistida - Flexão de quadril } \\
\text { resistida - Leg press - Extensão de quadril resistida Modulação } \\
\text { de tônus muscular hipotônico; } \\
\text { Escovação de Rood, em região de tibial anterior, com comando } \\
\text { verbal para dorsi e plantiflexão de tornozelo - Co-contração de } \\
\text { membros inferiores; } \\
\text { Apoio unipodal, abdução e extensão de quadril unilateralmente, } \\
\text { sobre cama elástica, apoiando-se em terapeuta. - Inclinação de } \\
\text { tronco sobre cama elástica, com terapeuta estabilizando na } \\
\text { altura do quadril - Agachamento até } 45 \text { o de flexão de quadril, } \\
\text { sobre Balance Dome, com apoio - Marcha sobre bases instáveis, } \\
\text { com uma e duas muletas; } \\
\text { Apoio unipodal no membro inferior direito, subindo e descendo } \\
\text { rampas e escadas; } \\
\text { - Realização de circuito com apoio em uma muleta, e segurando } \\
\text { uma boneca. }\end{array}$ & $\begin{array}{l}\text { A partir dos resultados obtidos, pode-se concluir que } \\
\text { o tratamento fisioterapêutico de uma gestante jovem } \\
\text { com sequelas de SGB foi efetivo para modulação do } \\
\text { tônus muscular, reeducação respiratória, melhora de } \\
\text { controle de tronco, aumento do equilíbrio estático, } \\
\text { redução das alterações posturais e diminuição da dor } \\
\text { lombo-pélvica, contribuindo para o parto natural. }\end{array}$ \\
\hline
\end{tabular}

A literatura descreve tratamentos fisioterapêuticos de maneiras distintas, sendo que cada fisioterapeuta com sua metodologia visa um objetivo em comum, que é o de promover e melhorar de forma geral a qualidade de vida dos pacientes acometidos pela Síndrome de Guillain-Barré. Carvalho et al. (2013) afirmam que exercícios cinesioterapêuticos associados com termoterapia; alongamentos globais; exercícios metabólicos; técnica de co-contração; hidroterapia; treino de transferências de peso e equilíbrio; e exercícios resistidos em cadeia cinética fechada e aberta promovem readequação a função de marcha e funcionalidade motora integral (força, tônus muscular e coordenação), respostas normais de MMSS, MMII e tronco, e maior independência para execução de habilidades funcionais e retorno as AVDs.

A cinesioterapia é o uso do movimento ou exercício utilizados como forma de terapia englobando recursos e técnicas variadas, incluindo mobilização ativa e passiva, reorganizando as possíveis alterações cinético-funcionais. Sua principal finalidade é a manutenção ou desenvolvimento livre para a sua função, e 
tem como efeitos principais a melhora da força motora e respiratória, resistência a fadiga, equilíbrio, coordenação motora, mobilidade e flexibilidade. A cinesioterapia utiliza exercícios de fortalecimento para o sistema muscular representando um papel essencial na fisioterapia e na reabilitação (GONÇALVES et al., 2016, citado por CONTTI, 2011).

Candotti et al. (2012) evidenciam co-contração e co-ativação como sendo a ocorrência de contração simultânea de dois ou mais músculos agonistas e antagonistas ao redor de uma articulação, objetivando a estabilização dinâmica e proteção articular, permitindo, também, ação muscular mais controlada de ajustes posturais.

Sá et al. (2015) recomenda que sejam realizados alongamentos e reforço da musculatura dos MMII utilizando o método Bobath: na execução de transferência e suporte de peso para os MMII, co-contração, método PNF: com iniciação rítmica, reversão dinâmica e de estabilização e estabilização rítmica. Visando uma maior progressão na força muscular de MMII, melhorando a ADM e o equilíbrio do paciente.

Peres et al. (2009) descrevem o método Bobath como uma técnica da fisioterapia onde o paciente realiza, mantém ou aprimora as suas funções de maneira mais coordenada, utilizando padrões que irão influenciar o tônus muscular, onde através dos pontos-chave, produz-se mudança do tônus muscular, influenciando no controle postural e no desempenho das atividades funcionais. Já o PNF é uma técnica tem como objetivo a facilitação, fortalecimento, ganho de controle e de coordenação dos movimentos. Ativa os proprioceptores que se localizam nas articulações, tendões e músculos, e consequentemente melhora a função motora (SILVA et al., 2017).

Em decorrência da desmielinização dos nervos motores, a SGB causa perda significativa de força, diminuição dos reflexos tendinosos, ocasiona atrofia muscular e déficit de equilíbrio, o que corrobora com os objetivos propostos neste estudo. Berchara (2008) define equilíbrio como uma reação automática capaz de recuperar a estabilidade após uma perturbação do centro de gravidade, e quando o corpo sofre perturbação no equilíbrio o controle postural entra em ação, sendo capaz de manter ou restaurar o equilíbrio em qualquer postura ou atividade.

Montini et al. (2016) defende que, além de alongamentos de membros inferiores, o treino de transferências com tábua; ortostatismo assistido em prancha; stand in table e barras paralelas (BP) com calha e tala extensora; trocas posturais tais como decúbito ventral (DV) com cunha, decúbito lateral esquerdo e direito; rolar; fortalecimento de musculatura de tronco e abdominal em sedestação, DV associado ao FES: todas essas técnicas podem melhorar de forma significativa o controle de tronco com dissociação de cintura escapular, ganho de força e melhora da coordenação em membros superiores, conseguindo manter-se em ortostatismo no stand in table e prancha ortostática sem complicações fisiológicas, permitindo ao paciente realizar posturas funcionais que haviam sido limitadas.

Não há literaturas, nem evidências que abordem ou comprovem o uso da aplicação de FES (Estimulação Elétrica Funcional) aplicada à musculatura de membros superiores ou inferiores visando melhorar a funcionalidade em pacientes com Síndrome de Guillain-Barré. A FES é um recurso 
eletroterapêutico utilizado de forma ampla na reabilitação de pacientes portadores de lesões neurológicas, desta forma faz-se necessário o desenvolvimento de estudos específicos na área.

Nascimento et al. (2012) observaram resultados favoráveis da fisioterapia na Síndrome de GuillainBarré após reabilitação de pacientes por um período de três meses, utilizando-se de técnicas como hidrocinesioterapia com diversos métodos de corrida através do comprimento da piscina associando alongamentos e exercícios globais e específicos das musculaturas dos membros superiores e dos membros inferiores, treinando transferências de peso, trabalhando o fortalecimento e a resistência muscular, assim como o uso de técnicas de relaxamento como tração cervical e Watsu. Após esse período, observou-se nesses pacientes recuperação total das amplitudes de movimentos e articulares, aumento da força muscular, das pressões respiratórias, melhora funcional sem sequelas e sem histórico de recidiva do quadro após obtenção de alta fisioterapêutica.

A hidrocinesioterapia utiliza os exercícios aquáticos para ajudar na reabilitação de várias patologias, pois, os movimentos na água são lentos e dão suporte às estruturas corporais permitindo maior mobilidade e, consequentemente, alongamentos mais eficientes. A imersão do paciente em água aquecida, favorece relaxamento muscular diminuindo dor e rigidez (HECKER et al., 2011).

Quadros et al. (2017) evidenciam progresso no tratamento fisioterapêutico realizado em uma gestante com Síndrome de Guillain-Barré, dando ênfase aos exercícios de sedestação como forma de reabilitação funcional; trabalhando transferências de peso com auxílio de bola suíça; fortalecimento muscular global; ajustes posturais passando de posição de sedestação para ortostatismo e vice-versa; e marcha em superfícies instáveis e realização de circuito com apoio. Concluindo que o tratamento fisioterapêutico de uma gestante jovem com sequelas de SGB foi efetivo para modulação do tônus muscular, reeducação respiratória, melhora de controle de tronco, aumento do equilíbrio estático, redução das alterações posturais e diminuição da dor lombo pélvica, contribuindo para o parto natural.

Leite et al. (2009) salientam que a bola suíça pode ser utilizada como instrumento terapêutico de grande auxílio no tratamento de problemas no equilíbrio, controle postural e força muscular, sendo utilizado tanto na avalição como no tratamento. Ao realizar exercícios em sedestação se obtém melhor uma melhor resposta adaptativa sendo necessário um feedback vestibular e proprioceptivo.

Trípolli et al. (2008) enfatizam que o controle postural é mantido através da orientação de inputs de sistemas somatossensoriais, visuais e vestibulares. As inabilidades em redistribuir o peso corpóreo limitam o paciente na realização de suas atividades de vida diárias. O reconhecimento e tratamento dos déficits de simetria e transferência é um aspecto importante na reabilitação. A transferência de peso corporal entre os membros inferiores é essencial para mobilidade funcional e equilíbrio normal do paciente favorecendo suas atividades de vida diária.

Dentre as literaturas onde se evidenciam as condutas terapêuticas supracitadas, as técnicas de alongamentos são benéficas para tratamento da SGB, pois visam corrigir as alterações musculoesqueléticas, proporcionado ganho de amplitude de movimento e flexibilidade, prevenção de contraturas e realizando 
ajustes posturais necessários. Di Alencar et al. (2010) definem o alongamento como uma manobra terapêutica utilizada para promover aumento da mobilidade e extensibilidade musculotendínea, do tecido conjuntivo, aumentar a ADM contribuindo para aumento da amplitude de movimento (ADM). Tendo por modalidade: alongamento estático, balístico e por facilitação neuromuscular proprioceptiva (FNP).

Embora as literaturas evidenciem que os resultados do tratamento geralmente sejam satisfatórios, algumas considerações tornam-se importantes, como estar alerta ao tempo de início do tratamento fisioterapêutico. (SÁ et al., 2015, citado por ORSINI et al., 2010) afirmam que o início tardio do tratamento pode prejudicar a evolução da reabilitação. É importante que o paciente procure tratamento fisioterapêutico o mais precoce possível após diagnóstico clínico da SGB. Sabe-se que o mais indicado para o processo de reabilitação da SGB é iniciar o tratamento fisioterapêutico durante as duas primeiras semanas após o diagnóstico da doença, período em que o próprio organismo começa o trabalho de remielinização dos nervos afetados, acelerando a recuperação e prevenindo mais sequelas.

\section{CONCLUSÃO}

A atuação fisioterapêutica é fundamental para o tratamento da SGB, atuando com técnicas de fisioterapia motora e respiratória, utilizando condutas de tratamento baseadas nas complicações e alterações cinético funcionais resultantes da Síndrome de Guillain-Barré. Os resultados adquiridos neste estudo demonstram que não existe um protocolo específico de tratamento na Síndrome de Guillain-Barré. Os dados obtidos não podem ser generalizados, embora cada profissional da fisioterapia possui sua metodologia fisioterapêutica onde tem como objetivo em comum proporcionar a independência e a melhora da qualidade de vida dos pacientes.

Considerando-se a crescente incidência dos casos de Síndrome de Guillain-Barré, assim como as sequelas incapacitantes, sugere-se a realização de mais estudos com estudos de caso que evidenciem sobre a patologia. Sendo assim, torna-se necessária a realização de estudos a respeito da atuação fisioterapêutica na Síndrome Guillain-Barré, a fim de se criar intervenções de reabilitação que visem melhorar a qualidade de vida desses pacientes.

\section{REFERÊNCIAS}

BECHARA, F. T.; SANTOS, S. M. S.. Efetividade de um programa fisioterapêutico para treino de equilíbrio em idosos. Revista Saúde e Pesquisa, v.1, n.1, 2008.

CANDOTTI, C. T.; CARVALHO, K. V.; LATORRE, M.; NOLL, M.; VARELA, M.. Revista Brasileira de Ciências do Esporte, Florianópolis, v.34, n.1, 2012.

CARVALHO, T. G. M. L. R.; LOPES, R. C.. A integralidade na atenção fisioterapêutica no paciente portador da síndrome de Guillain-Barré. Revista Biomotriz, v.7, n.2, 2013.

DI ALENCAR, T. A. M.; MATIAS K. F. S.. Princípios fisiológicos do aquecimento e alongamento muscular na atividade esportiva. Revista Brasileira de Medicina do Esporte, v.16, n.3, 2010.

FERRANINI, M. A. G.; SCATTOLIN, M. A. A.; RODRIGUES M. M.; REZENDE, M. H. F.; SANTOS, I. C. L.; IAZZETTI, A. V.. Síndrome de Guillain-Barré em associação temporal com a vacina influenza A. Revista Paulista Pediátrica, São Paulo, v.29, n.4, 2011.

GONÇALVES, C. A.; MONTERO, G. A.; FREITAS, N. A. S. B.. A importância da cinesioterapia no equilíbrio do idoso. Revista Discente da UNIABEU, Nilópolis, v.4, n.8, 2016.

HECKER, C. D.; MELO, C.; TOMAZONI, S.; MARTINS, R. A. B. L.; JUNIOR, E. C. P. L.. Análise dos efeitos da cinesioterapia e 
da hidrocinesioterapia sobre a qualidade de vida de pacientes com fibromialgia: um ensaio clínico randomizado.

Fisioterapia Movimento, Curitiba, v.24, n.1, 2011.

LABORATÓRIO HUMBERTO ABRÃO. Relação entre: Zika vírus, Síndrome de Guillain-Barré e microcefalia. Mangabeiras: Laboratório Humberto Abrão, 2015.

LEITE, N. N.; BORBA A. D. O.; SILVA M. J.; NASCIMENTO N. S.; SILVA N. A.; CONCEIÇÃO E. C. G.. Uso da bola terapêutica no equilíbrio estático e dinâmico de pacientes com hemiparesia. Fisioterapia Movimento, Curitiba, v.22, n.1, 2009.

MONTINI, F. T.; SOUZA, D. R.; RIBEIRO, F. Q.; BATTISTELLA, L. R.. Modelo intensivo de reabilitação na síndrome de GuillainBarré: um relato de caso. Acta Fisiátrica, São Paulo, v.23, n.1, p. 42-45, 2016.

MORAES, A.; CASAROLLI, A. C. G.; EBERHARDT, T. D.; HOFSTATTER, L. M.. Caracterização dos pacientes com Síndrome de Guillain-Barré internados em um hospital universitário. Revista Enfermagem Contemporânea, v.4, n.1, 2015.

NASCIMENTO, V. L. S.; BORBA, G. S.; LEITE C. M. B.; GARABINI, M. C.. Protocolo hidroterápico na Síndrome de Guillain-Barré: Estudo de caso. Revista Neurociências, Maceió, v.20, n.3, 2012.
PERES, L. W.; RUEDELL, A. M.; DIAMANTE, C.. Influência do conceito neuroevolutivo bobath no tônus e força muscular e atividades funcionais estáticas e dinâmicas em pacientes diparéticos espásticos após paralisia cerebral. Saúde Santa Maria, v.35, n.1, 2009.

QUADROS, L. R.; GRAVE, M. T. Q.. Fisioterapia em jovem gestante com sequelas pós-Síndrome de Guillain-Barré: relato de caso. Scientia Medica, v.27, n.1, 2017.

SÁ, B. P.; GRAVE, M. T. Q.; PÉRICO, E.; BOHRER, T. R. J.. Avaliação e tratamento de sequelas motoras pós-Síndrome de Guillain-Barré (SGB): Estudo de caso. Caderno Pedagógico Lajeado, v.12, n.3, 2015.

SILVA, I. A.; AMORIM, J. R.; CARVALHO, F. T.; MESQUITA, L. S. A.. Efeito de um protocolo de Facilitação Neuromuscular Proprioceptiva (FNP) no equilíbrio postural de idosas. Fisioterapia Pesquisa, v.24, n.1, p.62-67, 2017.

SOARES, J. L.; MONTEIRO, L. M.. A contribuição da fisioterapia na recuperação do paciente portador da Síndrome de Guillain-Barré: uma revisão integrativa. Revista Eletrônica Acervo Saúde, v.7, 2017.

TRÍPOLI, F.; MOREIRA S. R.; OBERG T. D.; LIMA N. M. F. V.. Tarefas orientadas e biofeedback: efeitos na transferência de peso em hemiparéticos. Acta Fisiátrica, São Paulo, v.15, n.4, p.220-224, 2008. 